\title{
TRANSLUCENCIA NUCAL
}

\section{Tamizaje de alteraciones cromosómicas fetales Trabajo presentado ante la Asamblea de la Sociedad de Cirugía de Bogotá.}

Camilo Hernández de Alba, MD. *

\section{Introducción}

En 1866, Langdon Down reportó que la piel de los recién nacidos afectados de trisomía 21 parecía muy larga para sus cuerpos. En 1876 Fraser y Mitchell publicaron una detallada descripción de 62 pacientes notando una asociación entre el trastorno y la edad materna avanzada. Se sabe ahora que el exceso de piel de los neonatos afectados por el síndrome de Down puede ser visualizada por ultrasonografía manifestándose como un incremento de la translucencia nucal (TN) en los primeros tres meses de embarazo. Así que la TN es una expresión fenotípica común en la mayoría de las trisomías, el Síndrome de Turner y las triploidías. Este estudio pretende evaluar la utilidad en nuestra población de la TN como método de tamizaje de alteraciones cromosómicas.

El tamizaje para trisomía 21 fue introducido al principio de la década de los setenta, cuando las técnicas de laboratorio se desarrollaron para la determinación del cariotipo fetal.

El primer intento se basó en la conocida asociación entre edad materna avanzada y alteraciones cromosómicas, ofreciendo amniocentesis a toda mujer mayor de 35 años. Con esta política de salud, se logra identificar tan solo al $25 \%$ de los fetos con síndrome de Down con una tasa de falsos positivos de $10 \%$. Después, en la década de los ochenta, se introduce la determinación de la $\alpha$-fetoproteína, el estriol no conjugado y la $\alpha$ gonadotropina coriónica, los cuales se integraron en un análisis de regresión multivariado difundido como "triple marcador", logrando índices de detección del $60 \%$ con falsos positivos del 5\%. En 1992 en el Harris Birth Rigth Reserch Center for Fetal Medicine en el King 's College Hospital de Londres, el doctor Kypros Nicolaides introduce la determinación sistemática de la TN por ecografía, entre las 10 y 14 semanas, con un

* Jefe del Servicio de Obstetricia. Hospital de San José punto de corte de $3 \mathrm{~mm}$ como límite superior normal, logrando índices de detección de $80 \%$ con una tasa de falsos positivos del $5 \%$.

\section{¿Qué es la translucencia nucal?}

Durante el segundo y tercer trimestres del embarazo, la acumulación anormal de líquido en la región retronucal ha sido clasificada como higroma quístico asociado a síndrome de Turner y edema nucal, los cuales tienen diversas etiologías que incluyen trisomías, defectos cardiovasculares y pulmonares, displasias esqueléticas, infección congénita y desórdenes hematológicos y metabólicos. Además, los fetos con TN anormal y cariotipo normal, tienen una menor tasa de sobrevida debido a la mayor incidencia de displasia esquelética, síndromes genéticos, cardiopatía e incremento de la mortalidad perinatal. En el primer trimestre, el término adecuado es el de TN, debido a que es la característica ecográfica que se observa, mientras que en el segundo trimestre cuando la TN se resuelve, el término es el de edema nucal.

\section{¿Cómo se determina?}

Se debe realizar una exploración ecográfica transabdominal, obteniendo una sección sagital media del feto para lograr la longitud cráneo-caudal. Se mide el máximo espesor entre la piel que recubre la columna cervical y el tejido celular subcutáneo. Es importante individualizar la membrana amniótica, la cual a esta edad gestacional no se ha fusionado con el corion y se encuentra flotando libremente en la cavidad amniótica. Para esto, se debe esperar a que el feto realice movimientos en forma espontánea o se hace uno de rebote con el transductor sobre el abdomen de la madre. Todo valor igual o superior a $3 \mathrm{~mm}$ se considera anormal (Figuras 1a y 1b). 


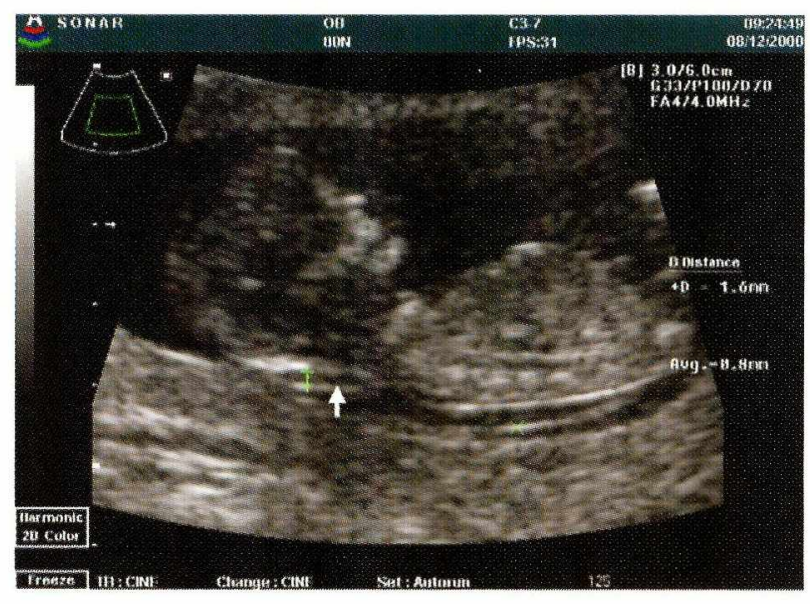

Figura Ia. Translucencia nucal en un feto cromosómicamente normal.

\section{Reproducibilidad}

Estudios realizados por el grupo de Nicolaides en 1995, demostraron que después de un entrenamiento adecuado, las diferencias intra e inter observador, se sitúan entre $0,54 \mathrm{~mm}$ y $0,62 \mathrm{~mm}$ respectivamente, permitiendo que la técnica pueda ser utilizada sólo en manos entrenadas.

\section{Translucencia nucal y defectos cromosómicos}

A principio de la década de los sesenta aparecieron varios reportes que incluían pequeñas series de embarazos de alto riesgo, demostrando una posible asociación entre alteraciones cromosómicas e incremento de la TN en el primer trimestre. Después, Nicolaides amplía el estudio a 1.273 embarazadas mayores de 35 años a quienes les realizó determinación de $\mathrm{TN}$ inmediatamente antes de la amniocentesis, encontrando un índice de detección del $80 \%$ para trisomía 21. Estos hallazgos permitieron concluir que la prevalencia de alteraciones cromosómicas depende de la edad materna y el espesor de la TN.

\section{Estudio del Frimley Park y St. Peters}

Habiendo demostrado la utilidad de la TN en pacientes seleccionadas de alto riesgo, el siguiente paso era confirmar su aplicación en embarazadas de bajo riesgo. Para esto, se diseñó el estudio del Frimley Park y St. Peters, dos hospitales del distrito general de Londres

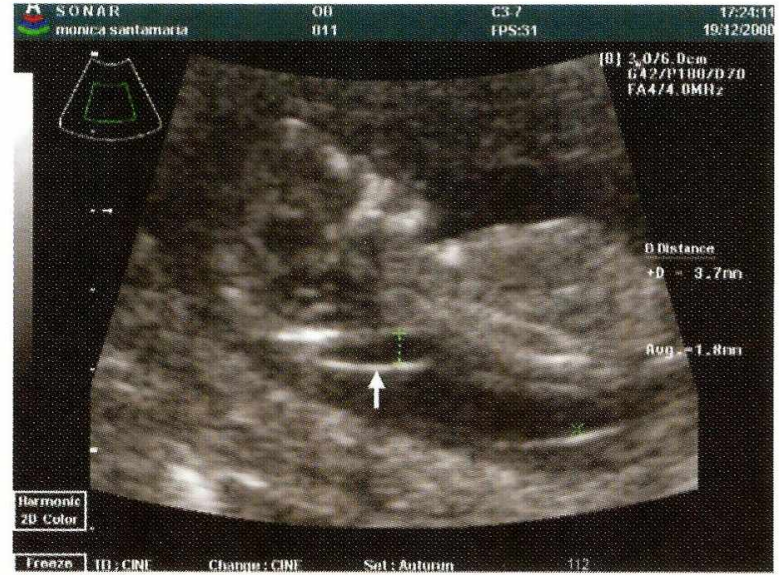

Figura Ib. Translucencia nucal patológica en un feto con trisomía 21 .

que atienden un promedio anual de 6.000 partos, y cuya política de salud era la de ofrecer amniocentesis a toda mujer mayor de 35 años o que tuviera antecedentes de hijos previos afectados. En un año se presentaron 11 casos de síndrome de Down de los cuales 10 pudieron ser detectados. Se decidió entonces incluir la determinación de la TN en forma sistemática entre las 10 y las 14 semanas. Tres meses después de esta nueva política de salud, se presentaron cuatro casos de síndrome de Down $\mathrm{y}$ todos fueron detectados.

\section{Fundación de Medicina Fetal}

Con el fin de unificar y auditar la determinación de la TN, surgió en Londres la Fundación de Medicina Fetal, como una entidad sin ánimo de lucro que propende por la educación médica y divulgación de la TN como método de tamizaje, mediante la realización de cursos y expedición del correspondiente certificado. En la actualidad existen en Inglaterra más de 30 centros certificados y por lo menos otros 20 a nivel mundial. El estudio inicial multicéntrico incluyó 42.619 pacientes de los cuales 20.543 fueron examinadas en Harris Birth Rigth y 22.076 en 19 hospitales del distrito general autorizados y certificados, logrando un índice de detección del $84 \%$ y una tasa de falsos positivos del $4,5 \%$.

Actualmente existen más de cien mil fetos sometidos a esta técnica de tamizaje, con un índice de detección superior al $80 \%$ y la tasa de falsos positivos menor de $5 \%$. 


\section{Hallazgos patológïcos}

Hyett y colaboradores en 1995, informaron la asociación entre el examen patológico del corazón y grandes vasos en fetos cromosómicamente anormales con un incremento de la TN. Las lesiones cardíacas más comunes en la trisomía 21 fueron los defectos septales auriculares y ventriculares, mientras que la trisomía 18 se asoció con defectos ventriculares septales y/o defectos polivalvulares. Además, el istmo aórtico se encontró más estrecho que en fetos normales, postulándose que esta disminución puede ser uno de los mecanismos responsables del incremento de la TN.

\section{Translucencia nucal en embarazos múltiples}

Durante los últimos veinte años, el promedio de edad materna y las técnicas de reproducción asistida se han incrementado, con el consecuente aumento en el número de embarazos múltiples con riesgo de alteraciones cromosómicas. En los embarazos múltiples, a diferencia de los únicos, el diagnóstico prenatal es difícil, debido a que los métodos de tamizaje bioquímico no son aplicables a los embarazos múltiples, y las técnicas invasivas conllevan mayor riesgo de pérdida fetal.

Pandya y colaboradores en 1995, examinan la TN en ocho pares de gemelos, en quienes el cariotipo demostrara al menos un gemelo afecto. Ocho fetos tuvieron trisomía 21 y dos trisomía 18. Se demostró así que en los embarazos múltiples, el tamizaje con base en la determinación de la TN y la edad materna tiene la misma sensibilidad que en los embarazos únicos.

\section{Translucencia nucal aumentada en fetos cromosómicamente normales}

Pandya y colaboradores reportan en 1994 y 1995 el resultado de 565 fetos cromosómicamente normales con TN aumentada. La prevalencia de defectos estructurales, en especial cardiacos, hernia diafragmática y alteraciones renales, fue mayor que en la población general.

La tasa de sobrevida, teniendo en cuenta muertes perinatales y terminación del embarazo por malforma- ciones, disminuyó de $97 \%$ con medidas de $3 \mathrm{~mm}$ a un $53 \%$ con medidas de 5 o más mm.

\section{Materiales y métodos}

\section{Estudio Hospital de San José}

En un período de 30 meses comprendido entre el 15 de febrero de 1998 y el 15 de agosto de 2000, se sometieron a tamizaje ecográfico para buscar alteraciones cromosómicas mediante la determinación de la TN, entre las 10 y las 14 semanas, un total de 508 fetos de mujeres embarazadas menores de 40 años, sin antecedentes de hijos previos afectos. La exploración ecográfica se realizó con transductores de 3,5 Mhz de alta definición multifrecuencia, por vía transabdominal con equipos de ultrasonido Toshiba ECCOCE o Medison 6000 color MT. El examinador siempre fue el mismo y se realizaron por lo menos dos determinaciones registrando siempre la mayor en $\mathrm{mm}$.

Los resultados de las medidas y los cálculos de riesgo se registraron en la base de datos fetal del programa del Dr. Kypros Nicolaides suministrado por la FUNDACIÓN DE MEDICINA FETAL DE LONDRES, a la cual se encuentra inscrito el Hospital de San José, previa certificación obtenida en el mes de junio de 1994. Los datos postparto del recién nacido fueron obtenidos mediante contacto telefónico con la paciente, información del médico tratante o correspondencia directa.

El riesgo individual para alteraciones cromosómicas se realizó con el software de la Fundación de Medicina Fetal, teniendo en cuenta como variables independientes la edad y el valor de la TN.

\section{Resultados}

- De las 508 pacientes sometidas a tamizaje, 29,7\% correspondía a población de más de 35 años.

- $5,5 \%$ de los fetos tuvieron TN por encima del percentil 95.

- $48,6 \%$ de los fetos tuvieron TN por encima de la mediana.

- $6,5 \%$ de las pacientes luego del cálculo computarizado del riesgo, tuvieron un riesgo de 1:300 o mayor. 
- La edad materna mínima fue de 17 años, la máxima de 40 y la media de 30 .

- A las pacientes cuyo riesgo integrado (obtenido por edad materna y valor de la translucencia nucal) arrojara un riesgo de 1:300 o más, se les aconsejó técnica invasiva para estudio de cariotipo fetal, mediante amniocentesis clásica realizada entre las 14 y 15 semanas (Tabla 1).

- Todas las pacientes fueron bien informadas del tipo de procedimiento invasivo (amniocentesis), los riesgos potenciales maternos y fetales derivados de la obtención de la muestra y del significado de un resultado positivo. Aceptaron la técnica y fueron llevadas, previo consentimiento informado, a amniocentesis genética a las 14 a 15 semanas. Las amniocentesis fueron realizadas por el mismo examinador con aguja $22 \mathrm{G}$, sin que se presentaran complicaciones maternas ni fetales asociadas a la técnica. Todos los líquidos amnióticos fueron analizados en el laboratorio de genética médica de la Fundación Gillow (Bogotá).

De los 508 fetos sometidos a tamizaje mediante la determinación de la $\mathrm{TN}$, se presentaron tres casos de alteraciones cromosómicas (Tabla 2), los cuales se ilustran en la Figura 2, con su correspondiente valor de $\mathrm{TN}$, edad materna, factor de riesgo y cariotipo. Se pre-

\section{Tabla 1. Resultados de 508 fetos sometidos a} tamizaje

\begin{tabular}{lr} 
Pacientes de 35 o más años & $29,7 \%$ \\
\hline Translucencia nucal > percentil 95 & $5,5 \%$ \\
\hline Translucencia nucal > media & $48,6 \%$ \\
\hline Riesgo I:300 o más & $6,5 \%$
\end{tabular}

Tabla 2. Alteraciones cromosómicas en 508 fetos

\begin{tabular}{cccccc} 
ID & CRL & TN & EM & RIESGO & CARIOTIPO \\
\hline I48 & 63 & 1,2 & 24 & $1: 13000$ & T 21 \\
\hline 538 & 58 & 5,0 & 31 & $1: 6$ & T 21 \\
\hline 432 & 73 & 9,0 & 37 & $1: 2$ & T 18 \\
\hline
\end{tabular}

CRL: Longitud cráneo caudal

TN: Translucencia nucal

EM: Edad materna sentó un solo caso de falso negativo en un feto con una TN de 2,1 mm y trisomía 21 documentada al nacimiento por cariotipo en sangre.

\section{Conclusiones}

1. La combinación de la edad materna y la TN determinada entre las 11 y 14 semanas ha demostrado ser el método más efectivo disponible en la actualidad para el tamizaje de alteraciones cromosómicas fetales con una sensibilidad para detección de trisomía 21 superior al $80 \%$.

2. Es el método ideal para ser utilizado en embarazos múltiples, debido a su comprobada eficacia y a la imposibilidad de usar el tamizaje bioquímico en dichos embarazos.

3. Es una técnica de fácil aplicación después de un entrenamiento adecuado y de bajo costo para el paciente, favoreciendo su cobertura a la población general.

4. La implementación de esta técnica no requiere la adquisición de nueva tecnología. Los equipos de ecografía disponibles en nuestro medio permiten su determinación en forma confiable.

5. Los fetos cromosómicamente normales con TN positiva, tienen un mayor riesgo de malformaciones fetales y morbilidad aumentada. La TN permite identificar a estos fetos en riesgo, e implementar medidas encaminadas a optimizar su cuidado prenatal.

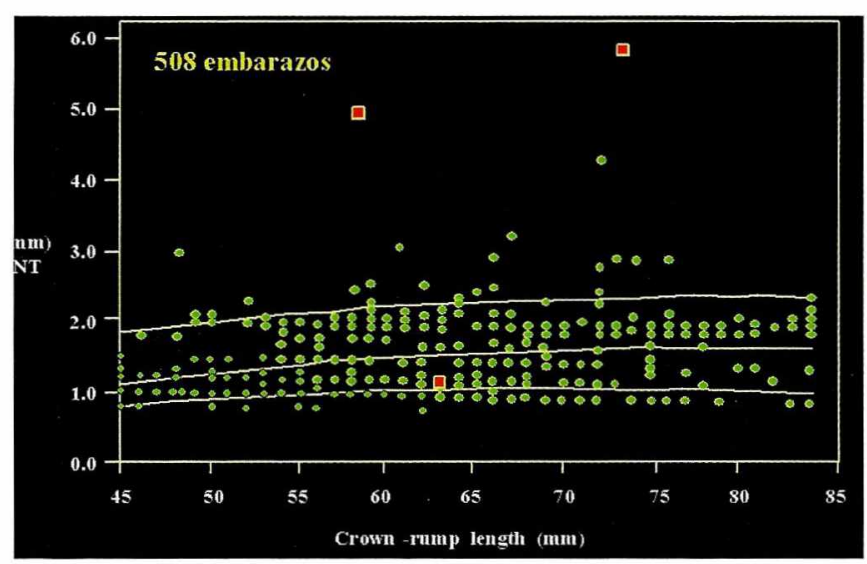

Figura 2. Ilustra la distribución de las translucencias nucales de acuerdo al CRL. En rojo los fetos con cariotipo anormal CRL: Longitud cráneo caudal 


\section{Bibliografía}

1. Nicolaides KH, Azar G, Byrne D, Mansur C, Marks K. Fetal nuchal translucency: ultrasound screening for chromosomal defects in first trimester of pregnancy. BMJ 1992, 304:867-9.

2. Pandya PP, Brizot ML, Altman D, Nicolaides KH: Reproducibility of measurement of fetal nuchal translucency thickness at 10-14 weeks gestation. Ultrasound Obstet Gynecol 1995; 5: 334-7.

3. Nicolaides KH, Azar G, Snijders RJM, Gosden CM. Fetal nuchal oedema: associated malformations and chromosomal defects. Fetal Diag Ther 1992, 7: 123-31.

4. Azar GB, Snijders RJM, Gosden C, Nicolaides KH. Fetal nuchal cystic higromata: associated malformations and chromosomal defects. Fetal Diag Ther 1991, 6:46-57.

5. Szabo J, Cellen J. Nuchal fluid acumulation in trisomy 21 detected by vaginosonography in first trimester. Lancet 1990, 336:1133.

6. Szabo J, Cellen J, Szemere G. First trimester ultrasound screening for fetal aneuploides in women over and less of 35 years of age. Ultrasound Obstet Gynecol 1995, 5: 161-3.
7. Hyett JA, Moscoso G, Nicolaides KH: First trimester nuchal translucency and cardiac septal defects in fetuses with trisomy 21. Am J Obstet Gynecol 1995; 172:1411-3.

8. Pandya PP, Brizot M, Snijders RJM, Kuhn P, Nicolaides KH. First trimester fetal nuchal translucency thickness and risk for fetal trisomies. Obstet Gynecol 1994, 84: 420-3.

9. Pandya PP, Kondylios A, Hilbert L, Snijders RJM, Nicolaides KH. Chromosomal defects and outcome in 1015 fetuses with increased nuchal translucency. Ultrasound Obstet Gynecol 1995,5: 15-9.

10. Snijders RJM, Holzgreve W, Cuckle H, Nicolaides KH. Maternal age-specific risk for trisomies at 9-14 weeks gestation. Prenat Diagn 1994, 14: 543-52.

11. Pandya PP, Goldberg H, Walton B, Riddle A, Shelley S, Snijders RJM, Nicolaides KH. The implementation of first trimester scanning at 10-13 weeks gestation and the measurement of fteal nuchal translucency thickness in two maternity units. Ultrasound Obste Gynecol 1995, 5: 20-5.

12. Pandya PP, Hilbert F, Snijders RJM, Nicolaides KH. Nuchal translucency thickness and crown-rump length in twin pregnancies with chromosomally abnormal fetuses. J Ultrasound Med 1995; 14:565-8.

\section{Fe de erratas. Revista Volumen 12 No. 1- 2003}

- En el índice debe incluirse el artículo que aparece en la página 25: "CITOLOGÍA ENDOMETRIAL DETOMA DIRECTA. ESTUDIO COMPARATIVO".

- En el índice, en vez de "EL ORIGEN DE LA VIDA, Dra Gladys León Sacedo", debe leerse: Dra Gladys León Salcedo, y en la página 34 dice: "Gladys León Sacedo M.D.” debe leerse: Dra. Gladys León Salcedo.

- Página 52: Repertorio Antaño, artículo ESPASMOS DE LA GLOTIS; debe agregarse: "Tomado del volumen 1 No 6 del 15 de marzo de 1910". 